\title{
Synthesis and Biological Activity of Azido Analogs of
}

\section{DMXAA for Use in Photoaffinity Labeling}

Brian D. Palmer, ${ }^{\dagger}$ Kimiora Henare,${ }^{\dagger}$ See-Tarn Woon, ${ }^{\dagger}$ Rachel Sutherland,${ }^{\dagger}$ Charu Reddy, ${ }^{\dagger}$ Liang-Chuan S. Wang, ${ }^{\dagger}$ Claudine Kieda, ${ }^{*}$ and Lai-Ming Ching ${ }^{* \dagger}$

Auckland Cancer Society Research Centre, Faculty of Medical and Health Sciences,

University of Auckland, Auckland, New Zealand, and Centre de Biophysique Moleculaire, UPR 4301 CNRS, Orleans Cedex, France

$\underline{\text { Table of Contents }}$

Combustion analysis data for new compounds: $\underline{\text { Page }}$

2 
$\underline{\text { Combustion Analyses }}$

\begin{tabular}{|c|l|c|c|c|c|c|c|}
\hline No & \multicolumn{1}{|c|}{ Formula } & \multicolumn{3}{|c|}{ Found } & \multicolumn{3}{c|}{ Calculated } \\
\hline & & C & H & N & C & H & N \\
\hline 2 & $\mathrm{C}_{15} \mathrm{H}_{19} \mathrm{~N}_{3} \mathrm{O}_{4}$ & 60.75 & 3.17 & 14.26 & 61.02 & 3.07 & 14.23 \\
\hline 3 & $\mathrm{C}_{16} \mathrm{H}_{11} \mathrm{~N}_{3} \mathrm{O}_{4} \cdot 0.5 \mathrm{H}_{2} \mathrm{O}$ & 60.71 & 3.66 & 12.83 & 60.38 & 3.80 & 13.20 \\
\hline 8 & $\mathrm{C}_{9} \mathrm{H}_{6} \mathrm{~N}_{2} \mathrm{O}_{4} \cdot 0.25 \mathrm{H}_{2} \mathrm{O}$ & 51.62 & 3.07 & 13.31 & 51.31 & 3.11 & 13.30 \\
\hline 9 & $\mathrm{C}_{8} \mathrm{H}_{8} \mathrm{~N}_{2} \mathrm{O}_{4}$ & 49.15 & 4.22 & 14.12 & 48.98 & 4.11 & 14.28 \\
\hline 10 & $\mathrm{C}_{8} \mathrm{H}_{6} \mathrm{INO}_{4} \cdot 0.25 \mathrm{MeOH}$ & 31.75 & 2.17 & 4.51 & 31.45 & 2.24 & 4.45 \\
\hline 11 & $\mathrm{C}_{16} \mathrm{H}_{13} \mathrm{NO}_{7}$ & 57.72 & 3.68 & 4.49 & 58.01 & 3.96 & 4.23 \\
\hline 13 & $\mathrm{C}_{16} \mathrm{H}_{13} \mathrm{NO}_{4}$ & 67.59 & 4.68 & 4.59 & 67.84 & 4.63 & 4.94 \\
\hline 16 & $\mathrm{C}_{9} \mathrm{H}_{6} \mathrm{~N}_{2} \mathrm{O}_{4} \cdot 0.125 \mathrm{H}_{2} \mathrm{O}$ & 51.82 & 3.15 & 13.33 & 51.87 & 3.02 & 13.44 \\
\hline 17 & $\mathrm{C}_{8} \mathrm{H}_{8} \mathrm{~N}_{2} \mathrm{O}_{4}$ & 49.35 & 4.22 & 14.28 & 48.98 & 4.11 & 14.28 \\
\hline 18 & $\mathrm{C}_{8} \mathrm{H}_{6} \mathrm{INO}_{4} \cdot 0.25 \mathrm{C}_{6} \mathrm{H}_{6}$ & 33.26 & 2.20 & 4.60 & 33.17 & 2.15 & 4.42 \\
\hline 19 & $\mathrm{C}_{16} \mathrm{H}_{13} \mathrm{NO}_{7}$ & 58.04 & 4.16 & 4.31 & 58.01 & 3.96 & 4.23 \\
\hline 21 & $\mathrm{C}_{16} \mathrm{H}_{13} \mathrm{NO}_{4} .0 .125 \mathrm{H}_{2} \mathrm{O}$ & 67.28 & 4.45 & 4.51 & 67.30 & 4.68 & 4.90 \\
\hline
\end{tabular}

